\title{
DUA FORMULA EKSPONENSIAL (OPERATOR LINEAR DARI SEMIGRUP)
}

\author{
L. Meisaroh ${ }^{1}$ \\ 1Program Studi D3 Teknologi Komputer, Fakultas IImu Terapan, Universitas Telkom \\ Jalan Telekomunikasi No.1, Terusan Buah Batu Bandung 40257, Indonesia \\ 1lisdameisaroh@telkomuniversity.ac.id
}

\begin{abstract}
Assumed $A$ is infinitesimal generator of $C_{0}$ semigroup $T(t)$ on $X$. This could be defined as $T(t)=e^{t A}$, applies if $A$ is a bounded linear operator. Not if $A$ is unbounded linear operator, then it will result in one possibility that show $T(t)$ could be represented as $e^{t A}$. This paper will discuss and detail the proof of the other two formulas that show $T(t)$ could be represented as $e^{t A .}$
\end{abstract}

Keywords : Infinitesimal Generator; Linear Operator; Exponential Formula.

\section{ABSTRAK}

Misalkan $A$ merupakan generator takhingga kecil dari $C_{0}$ semigrup $T(t)$ pada $X$. Hal ini dapat dinyatakan sebagai $T(t)=e^{t A}$, berlaku jika $A$ adalah operator linear terbatas. Lain halnya jika kasus $A$ merupakan operator linear tak terbatas, maka akan hanya memberikan satu kemungkinan yang menunjukkan bahwa $T(t)$ dapat direpresentasikan sebagai $e^{t A}$. Pada penelitian ini akan membahas dan mendetailkan pembuktian dua formula lainnya yang menunjukkan $T(t)$ dapat direpresentasikan sebagai $e^{t A}$.

Kata kunci : : Generator Takhingga Kecil; Operator Linear; Formula Eksponensial. 


\section{PENDAHULUAN}

Misalkan $A$ merupakan generator takhingga kecil dari $C_{0}$ semigrup $T(t)$ pada $X$. Maka berdasarkan [1] dapat dinyatakan sebagai

$$
T(t)=e^{t A}=\sum_{n=0}^{\infty} \frac{(t A)^{n}}{n !}
$$

Persamaan (1) berlaku jika $A$ adalah operator linear terbatas. Lain halnya jika kasus $A$ merupakan operator linear tak terbatas, maka hanya memberikan satu kemungkinan yang menunjukkan bahwa $T(t)$ dapat direpresentasikan sebagai $e^{t A}$. Pada penelitian ini akan membahas dan mendetailkan pembuktian dua formula lainnya yang menunjukkan $T(t)$ dapat direpresentasikan sebagai $e^{t A}$.

Pada formula eksponensial yang pertama, bentuk $T(t) x=\lim _{h \downarrow 0} e^{t A(h)} x$, didapat dari hasil turunan $T(t)$ pada saat $t=0$.

$$
\frac{d T(t)}{d t}=\left.A e^{A t} \rightarrow \frac{d T(t)}{d t}\right|_{t=0}=A e^{A(0)}=A
$$

Dapat dilihat, akan setara jika $h \downarrow 0$. Sedangkan untuk memahami formula yang kedua, yaitu bentuk $T(t) x=\lim _{n \rightarrow \infty}\left(I-\frac{t}{n} A\right)^{-n} x$, dapat dilihat dari konsep limit. Misalkan $y=\left(1-\frac{t}{n}\right)^{-n}$, maka $\ln y=$ $-n \ln \left(1-\frac{t}{n}\right)$.

$$
\lim _{n \rightarrow \infty} \ln y=\lim _{n \rightarrow \infty}\left(-n \ln \left(1-\frac{t}{n}\right)\right)=\lim _{n \rightarrow \infty} \frac{\ln \left(1-\frac{t}{n}\right)}{-1 / n}=\lim _{n \rightarrow \infty} \frac{\frac{1}{\left(1-\frac{t}{n}\right)}\left(t n^{-2}\right)}{n^{-2}}=\lim _{n \rightarrow \infty} \frac{t}{1-t / n}=t
$$

Sehingga didapatlah,

$$
\lim _{n \rightarrow \infty} y=\lim _{n \rightarrow \infty}\left(1-\frac{t}{n}\right)^{-n}=e^{t}
$$

Berikut adalah beberapa definisi dan teorema yang mendukung untuk pembuktian dua formula eksponensial yang akan dibahas pada penelitian ini.

Definisi 1. [1] Semigrup $T(t), 0 \leq t \leq \infty$, dari operator linear terbatas pada $X$ adalah semigrup kontinu kuat dari operator terbatas jika $\lim _{t \downarrow 0} T(t) x=0$ untuk setiap $x \in X$.

Teorema 2. [1] Misalkan $T(t)$ adalah $C_{o}$ semigrup. Terdapat konstanta $w \geq 0$ and $M \geq 1$ sehingga $\|T(t)\| \leq M e^{w t}$ untuk $0 \leq t<\infty$.

Teorema 3. [1] Misalkan $T(t)$ adalah $C_{o}$ semigrup dan misalkan $A$ adalah generator takhingga kecil, maka

a) Untuk $x \in X$,

$$
\lim _{h \rightarrow 0} \frac{1}{h} \int_{t}^{t+s} T(s) x d s=T(t) x
$$

b) Untuk $x \in X$,

$$
\int_{0}^{1} T(s) x d s \in D(A) \operatorname{dan} A\left(\int_{0}^{1} T(s) x d s\right)=T(t) x-x .
$$

c) Untuk $x \in D(A)$

$$
T(t) x \in D(A) \operatorname{dan} \frac{d}{d t} T(t) x=A T(t) x=T(t) A x .
$$

d) Untuk $x \in D(A)$,

$$
T(t) x-T(s) x=\int_{S}^{t} T(\tau) A x d \tau=\int_{S}^{t} A T(\tau) x d \tau .
$$


Teorema 4. [3] (Hille - Yoshida) Operator linear (tak terbatas) $A$ adalah generator takhingga kecil dari $C_{o}$ semigroup dari kontraksi $T(t), t \geq 0$ jika dan hanya jika

(i) $\quad A$ tertutup dan $\overline{D(A)}=X$.

(ii) Himpunan resolvent $\rho(A)$ dari $A$ memuat $\mathbb{R}^{+}$dan untuk setiap $\lambda>0$

$$
\|R(\lambda: A)\| \leq \frac{1}{\lambda}
$$

Lemma 5. [1] Misalkan $A$ memenuhi kondisi (i) dan (ii) dari Teorema 4 dan misalkan $R(\lambda: A)=$ $(\lambda I-A)^{-1}$. Maka $\lim _{\lambda \rightarrow \infty} \lambda R(\lambda: A) x=x$ untuk $x \in X$.

Teorema 6. [1] Misalkan $A$ memenuhi kondisi (i) dan (ii) dari Teorema 4. Jika $A_{\lambda}$ adalah approksimasi Yosida dari $A$, maka $A_{\lambda}$ adalah generator takhingga kecil dari semigrup kontinu seragam dari kontraksi $e^{t A_{\lambda}}$. Selanjutnya, untuk setiap $x \in X, \lambda, \mu>0$ akan berlaku

$$
\left\|e^{t A_{\lambda}}-e^{t A_{\mu}} x\right\| \leq t\left\|A_{\lambda} x-A_{\mu} x\right\|
$$

Lemma 7. [2] Misalkan $T(t)$ adalah $C_{o}$ semigroup. Akan ada konstanta $M \geq 1, \omega \geq 0$ yang memenuhi $\|T(t)\| \leq M e^{w t}$ untuk setiap $t \in \mathbb{R}^{+}$.

Persamaan 8. [1] Jika Operator linear $A$ adalah generator takhingga kecil dari $C_{o}$ semigroup $T(t)$ yang memenuhi $\|T(t)\| \leq M e^{w t}$, maka

$$
R(\lambda: A)^{(n)}=(-1)^{n} n ! R(\lambda: A)^{n+1} .
$$

\section{METODE PENELITIAN}

Metode penelitian yang dilakukan oleh penulis ini adalah studi literatur. Sumber utama yang dijadikan referensi penulis yaitu buku Semigroup of Linear Operators and Applications to Partial Differential Equations, karangan A. Pazy. Selain itu penulis juga mengumpulkan referensi yang relevan dengan sumber utama.

Penelitian ini dilakukan dengan tahapan pertama memahami alur pembuktian dua formula eksponensial. Setelah itu, mencari referensi lain untuk pendekatan pembuktian dua formula eksponensial tersebut. Dan terakhir mendetailkan pembuktiannya.

\section{HASIL DAN PEMBAHASAN}

\subsection{Formula Eksponensial Pertama}

Teorema 9. [1] Misalkan $T(t)$ dari $C_{0}$ semigrup $T(t)$ pada $X$. Jika $A(h) x=\frac{T(h) x-x}{h}$, maka $\forall x \in$ $X$, berlaku

$$
T(t) x=\lim _{h \downarrow 0} e^{t A(h)} x
$$


dan limitnya seragam di $t$ pada interval terbatas $[0, T]$.

Bukti:

Misalkan $T(t)$ adalah sebuah $C_{0}$ semigrup $T(t)$ pada $X$, sehingga berdasarkan Teorema 2, berlaku $\|T(t)\| \leq M e^{w t}, \omega>0$. Misalkan juga $A$ adalah generator tak hingga kecil dari $T(t)$. Karena $h>0$, maka $A(h)$ terbatas, sehingga $e^{t A(h)}$ well defined.

$$
A(h) x=\frac{T(h) x-x}{h} \rightarrow A(h)=\frac{T(h)-1}{h}
$$

Selanjutnya, karena $A(h)$ dan $T(t)$ saling menggantikan, begitupun dengan $e^{t A(h)}$ dan $T(t)$ sehingga berlaku

$$
\begin{aligned}
\left\|e^{t A(h)}\right\| & =\left\|\sum_{k=0}^{\infty} \frac{\left(t\left(\frac{T(h)-1}{h}\right)\right)^{k}}{k !}\right\|=\left\|\sum_{k=0}^{\infty} \frac{(t / h)^{k}(T(h)+(-1))^{k}}{k !}\right\| \leq\left\|\sum_{k=0}^{\infty} \frac{(t / h)^{k}(T(h))^{k}}{k !}\right\| \sum_{k=0}^{\infty} \frac{(-t / h)^{k}}{k !} \\
\left\|e^{t A(h)}\right\| & =e^{(-t / h)} \sum_{k=0}^{\infty}(t / h)^{k} \frac{\|T(h k)\|}{k !}
\end{aligned}
$$

Maka berdasarkan Teorema 2, didapat

$$
\left\|e^{t A(h)}\right\| \leq e^{(-t / h)} M e^{(t / h) e^{\omega h}}=M \exp \left\{-\frac{t}{h}+\frac{t}{h} e^{\omega h}\right\}=M \exp \left\{\frac{t}{h}\left(e^{\omega h}-1\right)\right\}
$$

Untuk $0<h<1$, berlaku $\left\|e^{t A(h)}\right\| \leq M e^{\left(t\left(e^{\omega}-1\right)\right)}$.

Ambil sebarang $x \in D(A)$, maka didapat

$$
\begin{aligned}
\frac{d}{d s}\left(e^{(t-s) A(h)} T(s) x\right) & =-A(h) e^{(t-s) A(h)} T(s) x+e^{(t-s) A(h)} A T(s) x \\
& =e^{(t-s) A(h)} T(s)(A x-A(h) x)
\end{aligned}
$$

Ambil juga sebarang $\varepsilon>0$, dan pilih $\delta=\frac{\varepsilon}{t M^{2} e^{t\left(e^{\omega}+\omega-1\right)}}$. Sehingga untuk $0<h<1$ dan $x \in D(A)$, $0<\|A x-A(h) x\|<\delta$, berlaku

$$
\left\|T(t) x-e^{t A(h)} x\right\|=\left\|\int_{0}^{1}\left(\frac{d}{d s}\left(e^{(t-s) A(h)} T(s) x\right)\right) d s\right\|
$$

Berdasarkan Teorema 3 dan 6, maka didapat

$$
\begin{aligned}
\left\|T(t) x-e^{t A(h)} x\right\| & =\left\|\int_{0}^{1} e^{(t-s) A(h)} T(s)(A x-A(h) x) d s\right\| \\
& =\int_{0}^{1}\left[\left\|e^{(t-s) A(h)}\right\|\|T(s)\|\|(A x-A(h) x)\|\right] d s \\
& \leq M e^{(t-s)\left(e^{\omega}-1\right)} \cdot M e^{\omega t} \cdot\|(A x-A(h) x)\|=t M^{2} e^{t\left(e^{\omega}+\omega-1\right)}\|(A x-A(h) x)\| \\
& <t M^{2} e^{t\left(e^{\omega}+\omega-1\right)} \cdot \frac{\varepsilon}{t M^{2} e^{t\left(e^{\omega}+\omega-1\right)}}=\varepsilon
\end{aligned}
$$




\subsection{Formula Eksponensial Kedua}

Teorema 10. [1] Misalkan $T(t)$ adalah sebuah $C_{0}$ semigrup pada $X$. Jika $A$ adalah generator takhingga kecil dari $T(t)$, maka

$$
T(t) x=\lim _{n \rightarrow \infty}\left(I-\frac{t}{n} A\right)^{-n} x=\lim _{n \rightarrow \infty}\left[\frac{n}{t} R\left(\frac{n}{t}: A\right)\right]^{n} x, x \in X
$$

dan limitnya seragam di $t$ pada sebarang interval terbatas.

Bukti:

Misalkan $T(t)$ adalah sebuah $C_{0}$ semigrup $T(t)$ pada $X$, sehingga berdasarkan Teorema 2, berlaku $\|T(t)\| \leq M e^{w t}, \omega>0$. Misalkan juga $A$ adalah generator tak hingga kecil dari $T(t)$. Jelaslah $\operatorname{Re} \lambda>\omega, R(\lambda: A)$ adalah analytics di $\lambda$ dan

$$
R(\lambda: A) x=\int_{0}^{\infty} e^{-\lambda s} T(s) x d s, x \in X .
$$

Maka untuk turunan ke- $n$ dari $R(\lambda: A) x$ adalah sebagai berikut.

$$
\begin{gathered}
\frac{d}{d \lambda} R(\lambda: A) x=\frac{d}{d \lambda}\left[\int_{0}^{\infty} e^{-\lambda s} T(s) x d s\right]=-\int_{0}^{\infty} s e^{-\lambda s} T(s) x d s \\
\frac{d^{2}}{d^{2} \lambda} R(\lambda: A) x=\frac{d^{2}}{d^{2} \lambda}\left[-\int_{0}^{\infty} s e^{-\lambda s} T(s) x d s\right]=\int_{0}^{\infty} s^{2} e^{-\lambda s} T(s) x d s \\
\frac{d^{3}}{d^{3} \lambda} R(\lambda: A) x=\frac{d^{3}}{d^{3} \lambda}\left[\int_{0}^{\infty} s^{2} e^{-\lambda s} T(s) x d s\right]=-\int_{0}^{\infty} s^{3} e^{-\lambda s} T(s) x d s \\
\vdots \quad \vdots \\
\frac{d^{n}}{d^{n} \lambda} R(\lambda: A) x=(-1)^{n} \int_{0}^{\infty} s^{n} e^{-\lambda s} T(s) x d s
\end{gathered}
$$

Dengan mensubtitusikan $s=v t \rightarrow d s=t d v$, dan mengambil nilai $\lambda=\frac{n}{t}$ sehingga didapat,

$$
\begin{aligned}
R\left(\frac{n}{t}: A\right)^{n} x & =(-1)^{n} \int_{0}^{\infty}(v t)^{n} e^{-\frac{n}{t}(v t)} T(v t) x t d v=(-1)^{n} t^{n+1} \int_{0}^{\infty} v^{n} e^{-n v} T(v t) x d v \\
& =(-1)^{n} t^{n+1} \int_{0}^{\infty}\left(v e^{-v}\right)^{n} T(t v) x d v
\end{aligned}
$$

Akan tetapi berdasarkan Persaman 8, didapatlah persamaan berikut ini.

$$
\begin{aligned}
(-1)^{n} n ! R\left(\frac{n}{t}: A\right)^{n+1} x & =(-1)^{n} t^{n+1} \int_{0}^{\infty}\left(v e^{-v}\right)^{n} T(t v) x d v \\
\frac{1}{n^{n+1}} \cdot \frac{n^{n+1}}{t^{n+1}} n ! R\left(\frac{n}{t}: A\right)^{n+1} x & =\int_{0}^{\infty}\left(v e^{-v}\right)^{n} T(t v) x d v \\
{\left[\frac{n}{t} R\left(\frac{n}{t}: A\right)\right]^{n+1} x } & =\frac{n^{n+1}}{n !} \int_{0}^{\infty}\left(v e^{-v}\right)^{n} T(t v) x d v
\end{aligned}
$$

Karena $\frac{n^{n+1}}{n !} \int_{0}^{\infty}\left(v e^{-v}\right)^{n} d v=1$, maka

$$
\left[\frac{n}{t} R\left(\frac{n}{t}: A\right)\right]^{n+1} x-T(t) x=\frac{n^{n+1}}{n !} \int_{0}^{\infty}\left(v e^{-v}\right)^{n}(T(t v) x-T(t) x) d v
$$

Ambil sebarang $\varepsilon>0$, pilih $0<a<1<b<\infty$ dimana $t \in\left[0, t_{0}\right]$, berlaku

$$
\|T(t v) x-T(t) x\|<\varepsilon, a \leq v \leq b
$$


Integral di ruas kanan pada persamaan (24) dipecah menjadi 3 interval, yaitu $I_{1}=[0, a], I_{2}=$ $[a, b]$, dan $I_{3}=[b, \infty)$. Maka hasil integral masing-masing interval adalah sebagai berikut.

$$
\begin{aligned}
& \left\|I_{1}\right\|=\frac{n^{n+1}}{n !} \int_{0}^{a}\left(v e^{-v}\right)^{n}\|T(t v) x-T(t) x\| d v \leq \frac{n^{n+1}}{n !}\left(a e^{-a}\right)^{n} \int_{0}^{a}\|T(t v) x-T(t) x\| d v \\
& \left\|I_{2}\right\|=\frac{n^{n+1}}{n !} \int_{a}^{b}\left(v e^{-v}\right)^{n}\|T(t v) x-T(t) x\| d v \leq \frac{n^{n+1}}{n !} \varepsilon \int_{a}^{b}\left(v e^{-v}\right) d v<\varepsilon \\
& \left\|I_{3}\right\|=\frac{n^{n+1}}{n !} \int_{b}^{\infty}\left(v e^{-v}\right)^{n}\|T(t v) x-T(t) x\| d v
\end{aligned}
$$

Dengan menggunakan fakta $v e^{-v}>0$, maka $v e^{-v}$ adalah fungsi monoton tak turun di $0 \leq v \leq$ 1 dan tak naik di $v \geq 1$. Selanjutnya, $v e^{-v}>e^{-1}$, untuk $v \neq 1$, maka $\left\|I_{1}\right\| \rightarrow 0$ seragam di $t \in\left[0, t_{0}\right]$ berlaku juga di $n \rightarrow \infty$. Pilih $n>\omega t$ di $I_{3}$. Dapat dilihat integral dari estimasi di $I_{3}$ konvergen dan $\left\|I_{3}\right\| \rightarrow 0$ seragam di $t \in\left[0, t_{0}\right]$ berlaku juga di $n \rightarrow \infty$. Akibatnya,

$$
\underset{n \rightarrow \infty}{\limsup }\left\|\left[\frac{n}{t} R\left(\frac{n}{t}: A\right)\right]^{n+1} x-T(t) x\right\|<\varepsilon .
$$

Dan karena $\varepsilon>0$ sebarang, maka $T(t) x=\lim _{n \rightarrow \infty}\left[\frac{n}{t} R\left(\frac{n}{t}: A\right)\right]^{n+1} x$.

Berdasarkan Lemma 5, maka didapat persamaan berikut ini.

$$
T(t) x=\lim _{n \rightarrow \infty}\left[\frac{n}{t} R\left(\frac{n}{t}: A\right)\right]^{n+1} x=\lim _{n \rightarrow \infty}\left[\frac{n}{t} R\left(\frac{n}{t}: A\right)\right] \underbrace{\lim _{n \rightarrow \infty}\left[\frac{n}{t} R\left(\frac{n}{t}: A\right)\right]^{n} x}_{x}=\lim _{n \rightarrow \infty}\left[\frac{n}{t} R\left(\frac{n}{t}: A\right)\right] x
$$

\section{KESIMPULAN}

Berdasarkan hasil dan pembahasan yang telah dilakukan, maka penelitian ini telah berhasil menjabarkan secara detail bukti dari kedua formula eksponensial. Untuk selanjutnya, formula eksponensial ini dapat menjadi pendekatan untuk mencari solusi dari persamaan diferensial.

\section{DAFTAR PUSTAKA}

[1]. Pazy, A., Semigroup of Linear Operators and Applications to Partial Differential Equations, New York, 1983, Springer-Verlag.

[2]. Goldstein, Jerome A, Semigroup of Linear Operators and Applications, New York, 1985, Oxford University Press.

[3]. Fetahu, Elona, On Semigroup of Linear Operators, http://mathematics.ceu.edu/ sites/ mathematics.ceu.hu/files/attachment/basicpage/29/elona-thesis.pdf, 2014, (diakses pada 19 April 2021). 